\section{Impact of the COVID-19 outbreak on adult out-of-hospital cardiac arrest outcomes in Daegu, South Korea: an observational study}

\author{
Jae Yun Ahn', Hyun Wook Ryoo', Jae Wan Cho', Jung Ho Kim², \\ Sang-Hun Lee ${ }^{3}$, Tae Chang Jang ${ }^{4}$
}

'Department of Emergency Medicine, Kyungpook National University School of Medicine, Daegu, Korea ${ }^{2}$ Department of Emergency Medicine, Yeungnam University Hospital, Yeungnam University College of Medicine, Daegu, Korea

${ }^{3}$ Department of Emergency Medicine, Dongsan Medical Center, Keimyung University School of Medicine, Daegu, Korea

${ }^{4}$ Department of Emergency Medicine, Daegu Catholic University School of Medicine, Daegu, Korea

Objective This study aimed to compare the outcomes of adult out-of-hospital cardiac arrest (OHCA) before and after the coronavirus disease 2019 (COVID-19) outbreak in a large metropolitan city.

Methods This before-and-after observational study used a prospective citywide OHCA registry. Adult patients with emergency medical service-treated OHCA, with presumed cardiac etiology, pre- and post-COVID-19 outbreak were enrolled. The study period spanned 2 months, starting from February 18, 2020. The control period was 2 months from February 18, 2019. The primary and secondary outcomes were good neurologic outcome and survival to hospital discharge, respectively. The association between the COVID-19 outbreak and OHCA outcomes was assessed using multivariable logistic regression analysis.

Results This study analyzed 297 OHCA patients (control period, 145; study period, 152). The bystander cardiopulmonary resuscitation rates were $64.8 \%$ and $60.5 \%$ during the control and study periods, respectively. Response and on-scene times increased by 2 minutes, supraglottic airway use increased by $35.6 \%$, and mechanical chest compression device use increased by $13 \%$ post-COVID-19 outbreak. Good neurologic outcome was significantly lower during the study period in overall OHCAs (adjusted odds ratio, 0.23; 95\% confidence interval, 0.05-0.98) and in witnessed OHCAs (adjusted odds ratio, 0.14; 95\% confidence interval, 0.02-0.90). No significant difference was found in the survival to hospital discharge of OHCA patients between the two periods.

Conclusion During the COVID-19 pandemic, the response and on-scene times were longer, and good neurologic outcome was significantly lower than that in the control period.

Keywords COVID-19; Out-of-hospital cardiac arrest; Cardiopulmonary resuscitation; Prognosis
elSSN: 2383-4625

Received: 5 January 2021

Accepted: 9 March 2021

Correspondence to: Hyun Wook Ryoo Department of Emergency Medicine, Kyungpook National University School of Medicine, 680 Gukchaebosang-ro, Jung-gu, Daegu 41944, Korea E-mail: ryoo@knu.ac.kr ORCID

https://orcid.org/0000-0002-1361-9887

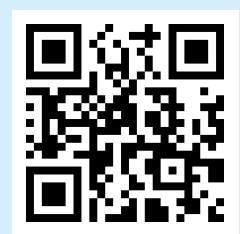

How to cite this article:

Ahn JY, Ryoo HW, Cho JW, Kim JH, Lee SH, Jang TC. Impact of the COVID-19 outbreak on adult out-of-hospital cardiac arrest outcomes in Daegu, South Korea: an observational study. Clin Exp Emerg Med 2021;8(2):137-144. https://doi. org/10.15441/ceem.21.008

This is an Open Access article distributed under the terms of the Creative Commons Attribution Non-Commercial License (https:// creativecommons.org/licenses/by-nc/4.0/). 


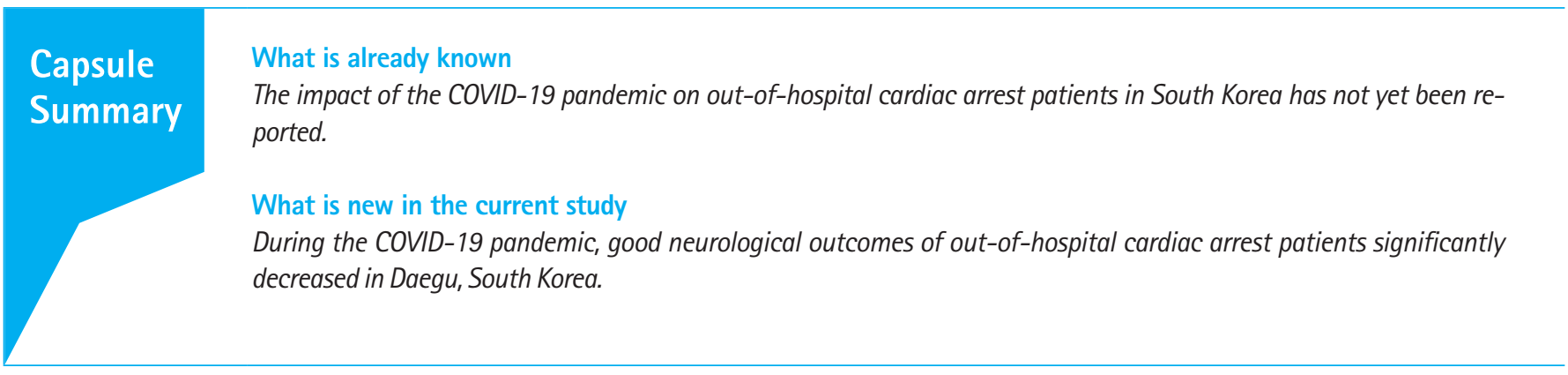

\section{INTRODUCTION}

The outbreak of coronavirus disease 2019 (COVID-19) has adversely impacted the resuscitation and outcome of out-of-hospital cardiac arrest (OHCA) patients. ${ }^{1,2}$ Compared with previous diseases caused by coronaviruses, such as the severe acute respiratory syndrome or the Middle East respiratory syndrome, COVID-19 has a higher transmission rate, and patients can either be asymptomatic or present with atypical symptoms including diarrhea., ${ }^{3.4}$

The high transmission rate of COVID-19 may hinder the execution of cardiopulmonary resuscitation (CPR) by laypersons due to fear of transmission. Infectious disease disasters, particularly COVID-19, may temporarily lead to emergency department (ED) closure due to the risk of infection in the ED and a lack of medical resources due to the overwhelming demand for emergency care. COVID-19 may present challenges in the initial response to emergency calls, in the provision of on-scene resuscitation, and in the integrated hospital treatment for OHCA patients whose COVID-19 status has not been confirmed. Nonetheless, the effects of the COVID-19 outbreak on OHCA patients in South Korea remain mostly unknown.

After Wuhan, Daegu was the second city in the world where the COVID-19 pandemic emerged, making it the COVID-19 epicenter of South Korea. An analysis of the key factors for resuscitation and the outcomes of OHCA patients during the COVID-19 pandemic is warranted to identify and revise any weak links in response to OHCAs. This study aimed to compare resuscitation and outcomes of OHCA patients between the control and study periods in a large metropolitan city that experienced the COVID-19 pandemic.

\section{METHODS}

\section{Study design and setting}

This study employed a before-and-after observational design using the Daegu Emergency Medical Service Registry, which is a prospective citywide OHCA registry that retrieves data from the emergency medical service (EMS) run sheet, dispatcher CPR registry, and hospital medical records according to the Utstein style guidelines. ${ }^{5}$ The study period spanned 2 months, starting from February 18, 2020, when the first COVID-19 case was detected in Daegu. The control period spanned from February 18, 2019 to April 17, 2019.

All emergency medical centers in this study were equipped with facilities, resources, and personnel that provide 24/7 advanced life support and postcardiac arrest care, including targeted temperature management (TM) and coronary reperfusion. The EMS team comprised a level-1 emergency medical technician (EMT) (similar to an EMT-intermediate in the United States) and a level-2 EMT (similar to an EMT-basic). A dual dispatch system was used for emergency calls involving cardiac arrest. ${ }^{5}$ Dispatcher-assisted CPR (DACPR) was attempted for OHCAs recognized by the emergency medical dispatcher. The emergency medical dispatcher provided compression only CPR instruction for OHCA patients with presumed cardiac etiology. The level-1 EMT performed advanced airway insertion and intravenous (IV) access under the medical direction. Shortly after the COVID-19 outbreak, regional emergency institutions and the local EMS teams collectively agreed to prioritize the use of supraglottic airway (SGA) for airway management and mechanical chest compression. The number of mechanical chest compression devices did not change between the control and study periods.

\section{COVID-19 in Daegu}

The first COVID-19 patient in Daegu, South Korea, was diagnosed on February 18, 2020, which was followed by an exponential increase in community cases. ${ }^{6}$ This resulted in Daegu experiencing a shortage of hospital beds, supplies, and healthcare workers, and this region was eventually declared a special disaster zone in South Korea. ${ }^{7}$ By April 18, 2020, a total of 6,830 COVID-19 cases were confirmed in Daegu, constituting 64.1\% $(6,830 / 10,653)$ of all COVID-19 cases in South Korea. 


\section{Study participants and data collection}

The study included all adults (aged 18 years or older) presenting with $\mathrm{OHCA}$, with presumed cardiac etiology, during the control and study periods. The exclusion criteria were patients who did not receive resuscitation attempts by EMS, arrests that were witnessed by EMTs, and patients who did not receive resuscitation attempts from the in-hospital medical team owing to a dead on arrival (DOA) status after arriving at the ED.

Data of the following variables were collected from the Daegu Emergency Medical Service Registry: demographics, including age, sex, and arrest location; factors associated with OHCA in the prehospital phase, including witness status, bystander CPR provision, first documented rhythm at the scene, prehospital advanced airway/mechanical chest compression device use, IV adrenaline use, response time (time interval from emergency call to EMS arrival at the scene), on-scene time (time interval from EMS arrival at the scene to departure for the hospital), and transport time (time interval from EMS departure to arrival at the ED); and factors associated with OHCA in the in hospital phase, including initial electrocardiogram rhythm at the ED, CPR duration, advanced airway management at the $E D, T M$, percutaneous coronary intervention, extracorporeal membrane oxygenation, survived event, survival to hospital discharge, and good neurologic outcome. Survived event and survival to hospital discharge definitions were based on the Utstein style guidelines. Good neurologic outcome was defined as Cerebral Performance Category 1 or 2.

\section{Outcome measurements}

The primary outcome was good neurologic outcome, while the secondary outcome was survival to hospital discharge.

\section{Statistical analyses}

All statistical analyses were conducted using IBM SPSS Statistics ver. 25.0 (IBM Corp., Armonk, NY, USA). The Mann-Whitney Utest was used for comparing continuous variables, while Pearson chi-square test was used for comparing categorical variables. To compare the clinical outcomes between the study and control periods, univariable and multivariable logistic regression analyses were performed and presented as an odds ratio (OR) with a 95\% confidence interval $(\mathrm{Cl})$. Multivariable logistic regression analysis was adjusted for analyzing age, sex, place of cardiac arrest, and initial documented rhythm. All statistical tests were two-tailed. $P$-values of $<0.05$ were considered statistically significant.

\section{Ethics statement}

This study was reviewed and approved by the institutional review board of Kyungpook National University Hospital (2016-03-027). The requirement for informed consent was waived by the institutional review board due to the retrospective nature of the study.

\section{RESULTS}

The numbers of EMS-assessed OHCA patients during the control and study periods were 209 and 219, respectively. Patients aged $<18$ years old or with noncardiac etiology, cardiac arrest witnessed by EMS, or those declared DOA were excluded. After exclusion, 145 and 152 OHCA patients were enrolled in the control and study groups, respectively. A flow diagram of the study participant selection is shown in Fig. 1.

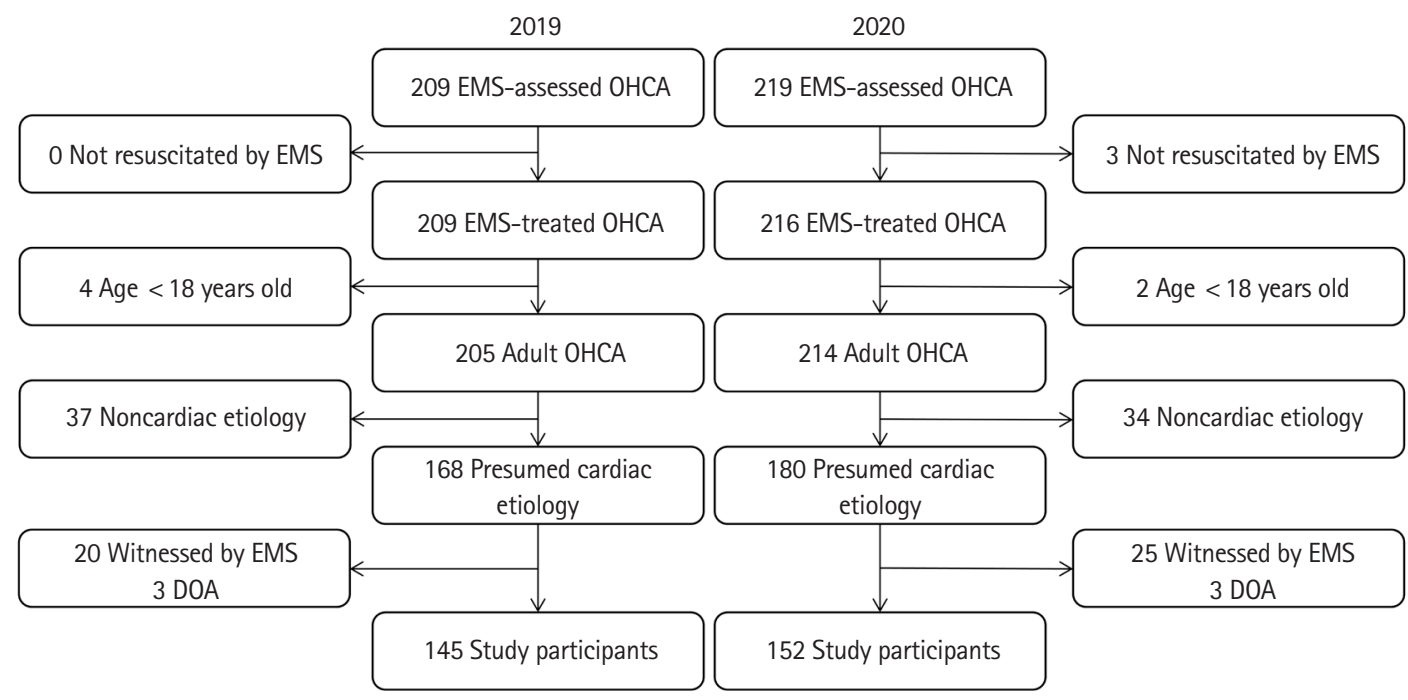

Fig. 1. Flow diagram of the study participant selection. EMS, emergency medical service; OHCA, out-of-hospital cardiac arrest; DOA, dead on arrival. 


\section{Comparison of general and resuscitative characteristics of OHCA patients}

Table 1 shows the baseline and resuscitative characteristics of OHCA patients in the control and study periods. Patients' median age was 76.0 years (interquartile range, $64.0-82.0$ years), and $65.0 \%$ of the participants were male. The proportions of cardiac arrests occurring at home were $83.6 \%$ and $77.2 \%$ in the study and control periods, respectively. From the control period to the study period, the bystander CPR rate decreased from $64.8 \%$ to $60.5 \%(P=0.444)$, while OHCA patients receiving CPR initiated by dispatcher instruction increased from $62.8 \%$ to $75.0 \%$. Furthermore, SGA use increased ( $40.7 \%$ to $76.3 \%$ ) while endotracheal intubation use decreased (51.7\% to 14.5\%) from the control period to the study period. The use of mechanical chest compression devices also significantly increased during the study period (74.5\% to $87.5 \%, P=0.004$ ) (Fig. 2). The response time was prolonged by 2 minutes, the first defibrillation of OHCA patients with initial shockable rhythm was delayed by 3 minutes, and the onscene time increased by 2 minutes in the study period compared with those in the control period, respectively $(P<0.001)$.

Table 1. Comparison of general and resuscitative characteristics of OHCA patients

\begin{tabular}{|c|c|c|c|c|}
\hline & Total $(n=297)$ & Control period $(n=145)$ & Study period $(n=152)$ & P-value \\
\hline Age (yr) & $76.0(64.0-82.0)$ & $74.0(61.5-82.0)$ & $76.0(66.0-81.8)$ & 0.159 \\
\hline Sex & & & & 0.432 \\
\hline Male & $193(65.0)$ & $91(62.8)$ & $102(67.1)$ & \\
\hline Female & $104(35.0)$ & $54(37.2)$ & $50(32.9)$ & \\
\hline Comorbidities & $251(84.5)$ & $124(85.5)$ & $127(83.6)$ & 0.640 \\
\hline Hypertension & $119(47.4)$ & $58(46.8)$ & $61(48.0)$ & 0.842 \\
\hline Diabetes mellitus & $98(39.0)$ & $52(41.9)$ & $46(36.2)$ & 0.353 \\
\hline Ischemic heart disease & $46(18.3)$ & $22(17.7)$ & $24(18.9)$ & 0.813 \\
\hline Stroke & $31(12.4)$ & $9(7.3)$ & $22(17.3)$ & 0.015 \\
\hline Location & & & & 0.170 \\
\hline Home & $239(80.5)$ & $112(77.2)$ & $127(83.6)$ & \\
\hline Public place & $58(19.5)$ & $33(22.8)$ & $25(16.4)$ & \\
\hline Dispatcher recognition & & & & 0.604 \\
\hline Yes & $213(71.7)$ & $106(73.1)$ & $107(70.4)$ & \\
\hline No & $84(28.3)$ & 39 (26.9) & $45(29.6)$ & \\
\hline Witnessed arrest & & & & 0.740 \\
\hline Yes & $134(45.1)$ & $64(44.1)$ & $70(46.1)$ & \\
\hline No & $163(54.9)$ & $81(55.9)$ & $82(53.9)$ & \\
\hline Bystander CPR & & & & 0.444 \\
\hline Yes & $186(62.6)$ & $94(64.8)$ & $92(60.5)$ & \\
\hline Before dispatcher instruction & $58(31.2)$ & $35(37.2)$ & $23(25.0)$ & \\
\hline After dispatcher instruction & $128(68.8)$ & $59(62.8)$ & $69(75.0)$ & \\
\hline No & $111(37.4)$ & $51(35.2)$ & $60(39.5)$ & \\
\hline Use of public-access AED by bystander & & & & 1.000 \\
\hline Yes & $4(1.3)$ & $2(1.4)$ & $2(1.3)$ & \\
\hline No & $293(98.7)$ & $143(98.6)$ & $150(98.7)$ & \\
\hline First documented rhythm & & & & 0.095 \\
\hline $\mathrm{VF} / \mathrm{pVT}$ & $44(14.8)$ & $24(16.6)$ & $20(13.2)$ & \\
\hline PEA & $61(20.5)$ & $36(24.8)$ & $25(16.4)$ & \\
\hline Asystole & $192(64.6)$ & $85(58.6)$ & $107(70.4)$ & \\
\hline Prehospital IV epinephrine use & & & & 0.639 \\
\hline Yes & $127(42.8)$ & $64(44.1)$ & $63(41.4)$ & \\
\hline No & $170(57.2)$ & $81(55.9)$ & $89(58.6)$ & \\
\hline Response time (min) & $7.0(5.0-9.0)$ & $6.0(5.0-7.0)$ & $8.0(7.0-11.0)$ & $<0.001$ \\
\hline On-scene time (min) & $19.0(15.0-22.0)$ & $17.0(14.0-20.5)$ & $19.0(17.0-23.0)$ & $<0.001$ \\
\hline Transport time (min) & $7.0(4.0-10.0)$ & $7.0(4.0-11.0)$ & $7.0(4.0-10.0)$ & 0.163 \\
\hline First defibrillation time (min) & $9.0(7.25-11.0)$ & $8.0(7.0-9.0)$ & $11.0(10.0-13.5)$ & $<0.001$ \\
\hline
\end{tabular}

Values are presented as median (interquartile range) or number (\%).

OHCA, out-of-hospital cardiac arrest; CPR, cardiopulmonary resuscitation; AED, automated external defibrillator; VF, ventricular fibrillation; pVT, pulseless ventricular tachycardia; PEA, pulseless electrical activity; IV, intravenous. 
$\square$ No $\square$ Endotracheal intubation $\square$ Supraglottic airway

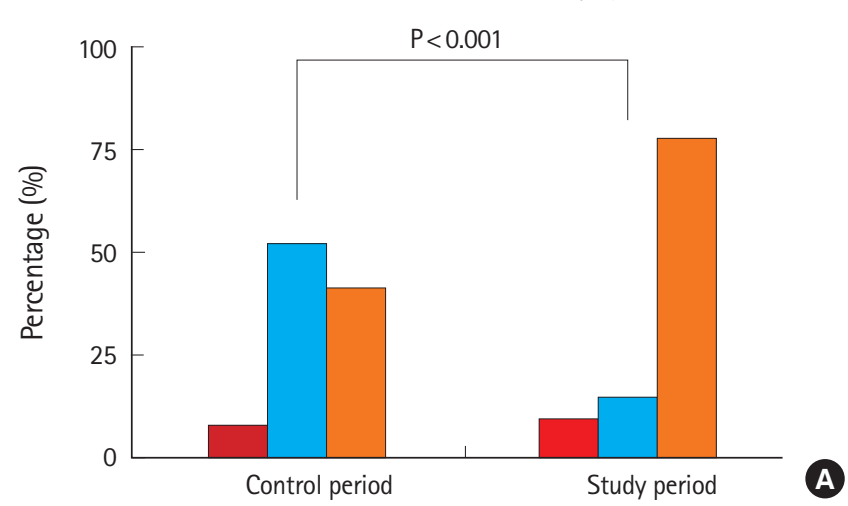

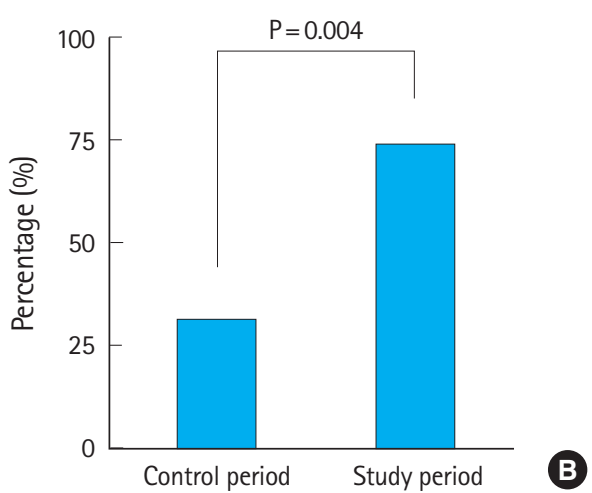

Fig. 2. Prehospital advanced airway management and mechanical chest compression device use in the control and study periods. (A) Prehospital advanced airway management and (B) prehospital mechanical chest compression device use.

Table 2. Bystander cardiopulmonary resuscitation performance according to witness status and location of cardiac arrest

\begin{tabular}{|c|c|c|c|c|}
\hline & Total $(n=297)$ & Control period $(n=145)$ & Study period $(n=152)$ & P-value \\
\hline \multicolumn{5}{|l|}{ Witness status } \\
\hline Witnessed & $94 / 134(70.1)$ & 46/64 (71.9) & $48 / 70(68.6)$ & 0.676 \\
\hline Before dispatcher instruction & $40(42.6)$ & $26(56.5)$ & 14 (29.2) & \\
\hline After dispatcher instruction & $54(57.4)$ & $20(43.5)$ & 34 (70.8) & \\
\hline Unwitnessed & $92 / 163(56.4)$ & 48/81 (59.3) & $44 / 82(53.7)$ & 0.471 \\
\hline Before dispatcher instruction & 18 (19.6) & $9(18.8)$ & $9(20.5)$ & \\
\hline After dispatcher instruction & $74(80.4)$ & 39 (81.3) & 35 (79.5) & \\
\hline \multicolumn{5}{|l|}{ Location } \\
\hline Home & 150/239 (62.8) & $73 / 112(65.2)$ & $77 / 127(60.6)$ & 0.777 \\
\hline Before dispatcher instruction & $39(26.0)$ & $23(31.5)$ & $16(20.8)$ & \\
\hline After dispatcher instruction & $111(74.0)$ & $50(68.5)$ & $61(79.2)$ & \\
\hline Public place & $36 / 58(62.1)$ & 21/33 (63.6) & $15 / 25(60.0)$ & 0.468 \\
\hline Before dispatcher instruction & $19(52.8)$ & $12(57.1)$ & $7(46.7)$ & \\
\hline After dispatcher instruction & $17(47.2)$ & $9(42.9)$ & $8(53.3)$ & \\
\hline \multicolumn{5}{|l|}{ Witness status and location } \\
\hline Witnessed, home & 77/106 (72.6) & $36 / 48(75.0)$ & 41/58 (70.7) & 0.620 \\
\hline Before dispatcher instruction & $33(42.9)$ & $20(55.6)$ & $13(31.7)$ & \\
\hline After dispatcher instruction & $44(57.1)$ & $16(44.4)$ & $28(68.3)$ & \\
\hline Witnessed, public & $17 / 28(60.7)$ & $10 / 16(62.5)$ & $7 / 12(58.3)$ & 1.000 \\
\hline Before dispatcher instruction & $7(41.2)$ & $6(60.0)$ & $1(14.3)$ & \\
\hline After dispatcher instruction & 10 (58.8) & $4(40.0)$ & $6(85.7)$ & \\
\hline Unwitnessed, home & 73/133 (54.9) & $37 / 64(57.8)$ & $36 / 69(52.2)$ & 0.514 \\
\hline Before dispatcher instruction & $6(8.2)$ & $3(8.1)$ & $3(8.3)$ & \\
\hline After dispatcher instruction & 67 (91.8) & $34(91.9)$ & $33(91.7)$ & \\
\hline Unwitnessed, public & $19 / 30(63.3)$ & $11 / 17(64.7)$ & $8 / 13(61.5)$ & 1.000 \\
\hline Before dispatcher instruction & $12(63.2)$ & $6(54.5)$ & $6(75.0)$ & \\
\hline After dispatcher instruction & $7(36.8)$ & $5(45.5)$ & $2(25.0)$ & \\
\hline
\end{tabular}

Values are presented as number (\%). The numerators and denominators indicate the number of bystander cardiopulmonary resuscitations and cardiac arrests, respectively.

\section{Bystander CPR performance according to witness status and location of cardiac arrest}

Table 2 shows the bystander CPR performance according to witness status and the location of cardiac arrest during the control and study periods. No significant differences were observed between the periods.

\section{Comparison of in hospital resuscitative characteristics and clinical outcomes of OHCA patients}

There were no significant differences in TTM $(2.8 \%$ to $0.7 \%, \mathrm{P}=$ $0.205)$ and percutaneous coronary intervention $(2.1 \%$ to $2.0 \%$, $\mathrm{P}=1.000$ ) between the control and study periods, respectively. Extracorporeal membrane oxygenation was not used during either period. The in-hospital CPR duration was similar for control 
Table 3. Comparison of in-hospital resuscitative characteristics and clinical outcomes of OHCA patients

\begin{tabular}{|c|c|c|c|c|}
\hline & Total $(n=297)$ & Control period $(n=145)$ & Study period $(n=152)$ & P-value \\
\hline Defibrillation & & & & 0.790 \\
\hline Yes & $28(9.4)$ & $13(9.0)$ & $15(9.9)$ & \\
\hline No & $269(90.6)$ & 132 (91.0) & 137 (90.1) & \\
\hline CPR duration (min) & $20.0(13.0-27.0)$ & $20.0(12.0-28.8)$ & $21.0(14.0-26.0)$ & 0.801 \\
\hline \multicolumn{5}{|l|}{ Outcome } \\
\hline ROSC & $33(11.1)$ & $25(17.2)$ & $8(5.3)$ & 0.001 \\
\hline Survival to hospital discharge & $22(7.4)$ & $15(10.3)$ & $7(4.6)$ & 0.059 \\
\hline Good neurologic outcome & $19(6.4)$ & $14(9.7)$ & $5(3.3)$ & 0.025 \\
\hline
\end{tabular}

Values are presented as number (\%) or median (interquartile range).

OHCA, out-of-hospital cardiac arrest; CPR, cardiopulmonary resuscitation; ROSC, prehospital return of spontaneous circulation.

Table 4. Logistic regression analysis of survival outcomes according to the COVID-19 outbreak

\begin{tabular}{|c|c|c|c|c|c|c|}
\hline & \multicolumn{2}{|c|}{ Good neurologic outcome } & \multicolumn{2}{|c|}{ Survival to hospital discharge } & \multicolumn{2}{|c|}{ ROSC } \\
\hline & OR $(95 \% \mathrm{Cl})$ & $\mathrm{aOR}^{\mathrm{a})}(95 \% \mathrm{Cl})$ & OR $(95 \% \mathrm{Cl})$ & $\mathrm{aOR}^{\mathrm{a})}(95 \% \mathrm{Cl})$ & OR $(95 \% \mathrm{Cl})$ & $\mathrm{aOR}^{\mathrm{a})}(95 \% \mathrm{Cl})$ \\
\hline \multicolumn{7}{|l|}{ Overall } \\
\hline Study period & $0.32(0.11-0.91)$ & $0.23(0.05-0.98)$ & $0.42(0.17-1.06)$ & $0.42(0.12-1.41)$ & $0.27(0.12-0.61)$ & $0.25(0.09-0.68)$ \\
\hline \multicolumn{7}{|l|}{ Witnessed arrest } \\
\hline \multicolumn{7}{|c|}{ Unwitnessed arrest } \\
\hline Control period & 1.00 & 1.00 & 1.00 & 1.00 & 1.00 & 1.00 \\
\hline Study period & $0.99(0.06-16.06)$ & $0.43(0.01-14.13)$ & $0.49(0.04-5.49)$ & $0.35(0.02-5.56)$ & $0.47(0.11-1.97)$ & $0.55(0.13-2.41)$ \\
\hline
\end{tabular}

COVID-19, coronavirus disease 2019; OR, odds ratio; aOR, adjusted odds ratio; $\mathrm{Cl}$, confidence interval; ROSC, prehospital return of spontaneous circulation.

${ }^{a)}$ Adjusted for age, sex, location of cardiac arrest, and first documented rhythm.

and study groups (20.0 vs. 21.0 minutes), respectively. Good neurologic outcome, survival to hospital discharge, and survived events decreased from $9.7 \%$ to $3.3 \%(P=0.025)$, from $10.3 \%$ to $4.6 \%$ $(P=0.059)$, and from $17.2 \%$ to $5.3 \%(P=0.001)$, in the control and study periods, respectively. These results show that the study period had poorer clinical outcomes than the control period (Table 3).

\section{Logistic regression analyses of survival outcomes pre- and post-COVID-19 outbreak}

Table 4 shows the results of univariable and multivariable logistic regression analyses for survival outcomes pre- and post-COVID-19 outbreak. Univariable analysis revealed significantly lower good neurologic outcomes $(\mathrm{OR}, 0.32 ; 95 \% \mathrm{Cl}, 0.11-0.91)$ and survived events $(\mathrm{OR}, 0.27 ; 95 \% \mathrm{Cl}, 0.12-0.61)$ in the study period than in the control period. After adjusting for potential confounders, good neurologic outcomes (adjusted OR [aOR], 0.23; 95\% Cl, 0.050.98) and survived events $(a O R, 0.25 ; 95 \% \mathrm{Cl}, 0.09-0.68)$ were significantly lower in the study period than in the control period. No significant differences in survival to hospital discharge were observed in the univariable and multivariable analyses. For witnessed arrest, in multivariable logistic regression analysis, good neurologic outcomes ( $\mathrm{aOR}, 0.14 ; 95 \% \mathrm{Cl}, 0.02-0.90)$ and survived events $(\mathrm{aOR}, 0.11 ; 95 \% \mathrm{Cl}, 0.03-0.52)$ were significantly lower in the study period than in the control period, but no significant difference was found in survival to hospital discharge $(a O R, 0.40$; 95\% $\mathrm{Cl}, 0.09-1.71)$.

\section{DISCUSSION}

An approximately 4.8\% increase in the incidence of EMS-assessed OHCA was observed during the COVID-19 pandemic in Daegu, South Korea, which was less extreme than that observed in Italy and Paris. ${ }^{1,2}$ Although many patients who needed treatment avoided hospital visits out of fear of exposure to COVID-19, only a slight increase was observed in the number of OHCA patients, which may have been due to the absence of lockdown, quarantine, or travel restriction measures in Daegu.

The rate of bystander CPR decreased by $4.3 \%$ following the COVID-19 outbreak, but this was not statistically significant. The high rate of human to human transmission of COVID-19 may negatively influence the willingness of a layperson to perform bystander CPR. ${ }^{8}$ The tendency to avoid outings may be related to 
the increased proportion of at-home cardiac arrests. Given that most bystanders of at-home OHCA are family members who may be more aware of the patient's preexisting conditions, they would be less likely to avoid performing bystander CPR. An increased rate of CPR performed upon dispatcher instruction was observed, which seems to have contributed to maintaining the bystander CPR rate. The compression only CPR for cardiac arrests may have contributed to an increased willingness to perform bystander CPR. The time of initial chest compression delivery, based on the dispatcher instructions in DACPR, was not considered in this study. Although the recommendation is to start chest compressions within 120 seconds for bystander CPR, ${ }^{9}$ the need for the dispatch center to assess COVID-19-related symptoms in patients with cardiac arrest may delay the onset of initial chest compressions. Further research should examine whether a comparable level of DACPR can be provided during an infectious disease outbreak relative to one before the outbreak.

The delay in first defibrillation time for OHCA patients with shockable rhythm can be attributed to the increase in response time. During the COVID-19 outbreak, a minimum standard of level $D$ personal protective equipment (PPE) is recommended when in contact with an OHCA patient with an unconfirmed COVID-19 status. The associated delay is inevitable when implementing precautionary measures to minimize COVID-19 exposure and transmission from OHCA patients. The surge in COVID-19 cases may have led to increased response times in certain regions due to ambulance shortages. Longer response times negatively impact good neurologic outcomes and survival to hospital discharge, but the provision of bystander CPR may offset some of the negative effects of longer response times. ${ }^{10}$ Therefore, emphasis on bystander CPR during COVID-19 outbreaks should be highlighted as well as the critical role of DACPR in initiating and maintaining bystander CPR.

The COVID-19 outbreak in Daegu occurred before the release of the COVID-19 guidelines of the American Heart Association and European Resuscitation Council on the use of advanced airway and mechanical chest compression devices in COVID-19 patients. ${ }^{11,12}$ Due to the local response protocol encouraging the use of SGA rather than endotracheal intubation, the use of the former increased while the latter decreased. The use of mechanical chest compression devices was suggested to minimize the risk of infection by limiting resuscitation and to overcome the challenges of performing chest compressions for extended periods while wearing PPE. This approach should be encouraged as early as possible in field resuscitation. The increased use of SGA and mechanical chest compression devices highlights the benefits of implementing local response protocols for OHCA. In preparation for a second wave of COVID-19, PPE, equipment for advanced airway management, and mechanical chest compression devices should be provided, along with training for their efficient use.

The World Health Organization recommends that the administration of aerosol generating procedures, including intubation or CPR by healthcare workers, occurs in isolated negative pressure rooms (or a facility in which a ventilation rate of $160 \mathrm{~L} / \mathrm{s} /$ patient is possible by natural ventilation) where air exchange can occur at least 12 times per hour to prevent transmission from patients diagnosed with or suspected of having COVID-19. ${ }^{13}$ During the surge in patients following the first COVID-19 case, the main issue in Daegu was a severe shortage of negative pressure rooms in EDs rather than a PPE shortage. Given that many suspected COVID-19 patients were required to stay in negative pressure rooms until their COVID-19 test results became available, the number of negative pressure rooms was frequently inadequate. Allocating dedicated rooms for OHCA patients in existing facilities was challenging, especially in an environment where the use of negative pressure rooms was suddenly increasing. During the study period, the on-scene time increased by 2 minutes without field CPR protocol change for EMS rescuers. When the COVID-19 outbreak started, a shortage of healthcare workers occurred because some underwent self-quarantine after being exposed to COVID-19 patients, and some EDs were temporarily closed due to the presence of COVID-19 patients; indeed, temporary ED closures recurred in several hospitals. ${ }^{14}$ These conditions likely exacerbated the delay in on-scene wait times by limiting the hospitals to which EMT rescuers had access.

To overcome such challenges, administering advanced life support in public ambulances outside the ED should be considered first. Several OHCA patients in Daegu received CPR in a public ambulance outside an ED after the COVID-19 outbreak. If a mechanical chest compression device is available to enable chest compressions, SGA and IV access can be properly executed in an ambulance, a useful alternative to negative pressure rooms. Second, establishing a separate resuscitation room outdoors where natural ventilation is available should be considered to prevent contamination inside the ED when performing aerosol generating procedures and create an isolated area where resuscitation can occur. The $160 \mathrm{~L} / \mathrm{s}$ ventilation rate via natural ventilation, recommended by the World Health Organization, can be attained by establishing an outdoor resuscitation room despite the absence of negative pressure infrastructure. ${ }^{15}$ Additional space and budget to install negative pressure tents or infrastructure outside the ED may be required. Finally, applying the termination of resuscitation (TOR) rule to OHCA can be considered during an infectious disease outbreak. Currently, in South Korea, applying field TOR to 
OHCA patients is impossible. Most victims without signs of irreversible death must be transferred to a hospital, where TOR must be authorized by a physician. Although no accounts of TOR in Daegu occurred during the COVID-19 outbreak, a discussion on the TOR rule for OHCA is required in the event of future infectious disease outbreaks.

This study has several limitations. First, the results may not be generalizable owing to the small number of OHCA patients studied in a single metropolitan city. Second, this study aimed to compare the changes in characteristics and prognoses of OHCA during a two-month COVID-19 outbreak. Due to the focus on shortterm changes in OHCA patient characteristics, long-term changes in CPR trends or prognoses according to the infectious disease outbreak were not assessed.

In conclusion, good neurologic outcomes of overall and witnessed OHCA showed a significant decrease over the study period. The response and on-scene times increased significantly during the study period. To provide care for OHCA patients during the COVID-19 outbreak, strategies to resolve negative pressure room shortages in hospitals must be implemented.

\section{CONFLICT OF INTEREST}

No potential conflict of interest relevant to this article was reported.

\section{REFERENCES}

1. Baldi E, Sechi GM, Mare C, et al. Out-of-hospital cardiac arrest during the Covid-19 outbreak in Italy. N Engl J Med 2020; 383:496-8.

2. Marijon $E_{1}$ Karam $N$, Jost $D$, et al. Out-of-hospital cardiac arrest during the COVID-19 pandemic in Paris, France: a population-based, observational study. Lancet Public Health 2020; 5:e437-43.

3. Rothan HA, Byrareddy SN. The epidemiology and pathogenesis of coronavirus disease (COVID-19) outbreak. J Autoimmun 2020;109:102433.

4. Kim GU, Kim MJ, Ra SH, et al. Clinical characteristics of asymptomatic and symptomatic patients with mild COVID-19. Clin Microbiol Infect 2020;26:948.

5. Kim JH, Ryoo HW, Kim JY, et al. Application of a dual-dispatch system for out-of-hospital cardiac arrest patients: will more hands save more lives? J Korean Med Sci 2019;34:e141.

6. Kim SW, Lee KS, Kim K, Lee JJ, Kim JY; Daegu Medical Associ- ation. A brief telephone severity scoring system and therapeutic living centers solved acute hospital-bed shortage during the COVID-19 outbreak in Daegu, Korea. J Korean Med Sci 2020;35:e152.

7. Kim JH, An JA, Min PK, Bitton A, Gawande AA. How South Korea responded to the Covid-19 outbreak in Daegu. NEJM Catal Innov Care Deliv 2020;1. https://doi.org/10.1056/CAT. 20.0159

8. Christian MD, Loutfy M, McDonald LC, et al. Possible SARS coronavirus transmission during cardiopulmonary resuscitation. Emerg Infect Dis 2004;10:287-93.

9. American Heart Association. CPR \&t first aid: emergency cardiovascular care. Telephone CPR (T-CPR) program recommendations and performance measures [Internet]. Dallas, TX: American Heart Association; 2018 [cited 2020 Aug 10]. Available from: https://ahainstructornetwork.americanheart.org/idc/ groups/heart-public/@wcm/@ecc/documents/downloadable/ucm_493303.pdf.

10. Moon S, Ryoo HW, Ahn JY, Lee DE, Shin SD, Park JH. Association of response time interval with neurological outcomes after out-of-hospital cardiac arrest according to bystander CPR. Am J Emerg Med 2020;38:1760-6.

11. Edelson DP, Sasson C, Chan PS, et al. Interim guidance for basic and advanced life support in adults, children, and neonates with suspected or confirmed COVID-19: from the Emergency Cardiovascular Care Committee and Get With The GuidelinesResuscitation Adult and Pediatric Task Forces of the American Heart Association. Circulation 2020;141:e933-43.

12. Nolan JP, Monsieurs KG, Bossaert $L$, et al. European Resuscitation Council COVID-19 guidelines executive summary. Resuscitation 2020;153:45-55.

13. World Health Organization. Clinical management of severe acute respiratory infection when novel coronavirus (2019$\mathrm{nCoV}$ ) infection is suspected: interim guidance, 28 January 2020 [Internet]. Geneva: World Health Organization; 2020 [cited 2020 Aug 10]. Available from: https://apps.who.int/iris/ handle/10665/330893.

14. Chung HS, Lee DE, Kim JK, et al. Revised triage and surveillance protocols for temporary emergency department closures in tertiary hospitals as a response to COVID-19 crisis in Daegu metropolitan city. J Korean Med Sci 2020;35:e189.

15. World Health Organization. Natural ventilation for infection control in health-care settings [Internet]. Geneva: World Health Organization; 2009 [cited 2020 Aug 10]. https://www.who.int/ water_sanitation_health/publications/natural_ventilation.pdf. 